\title{
Evolução da massa corporal magra após 12 meses da cirurgia bariátrica
}

\author{
Lean body mass changes within 12 \\ months of bariatric surgery
}

Selma Freire de Carvalho da CUNHA'

Maísa SANCHES ${ }^{1}$

Angélica FARIA

José Ernesto dos SANTOS 1

Carla Barbosa NONINO-BORGES

RE S U M O

\section{Objetivo}

O objetivo da pesquisa foi determinar a perda de massa corporal magra em pacientes após cirurgia bariátrica.

\section{Métodos}

O estudo retrospectivo foi conduzido com 17 prontuários de mulheres obesas submetidas à Derivação Gástrica em $Y$ de Roux com anel de contenção gástrica, incluindo dados obtidos no período pré-operatório imediato e no $1^{\circ}, 3^{\circ}, 6^{\circ}$ e $12^{\circ}$ meses após a cirurgia. Os dados obtidos no prontuário inclúram a idade, medidas de peso, de altura e massa corporal magra e gorda, calculados pela impedância bioelétrica.

\section{Resultados}

A média de idade das pacientes foi de $43,1, D P=7,7$ anos e durante o seguimento houve diminuição significativa do índice de massa corporal $\left[51,2(40,2-74,1)\right.$ para $\left.33,7(24,8-53,4) \mathrm{kg} / \mathrm{m}^{2}\right]$ e da massa corporal gorda $[67,5$ $(51,2-67,4)$ para $32,1(16,4-61,9) \mathrm{kg}]$ em 12 meses de seguimento. No primeiro mês após a cirurgia, houve diminuição da massa corporal magra ( $M=65,3, D P=7,6$ para $M=59,7, D P=8,1 \mathrm{~kg}$ ), que representou $8,5 \%$ em relação aos valores iniciais, sendo que a partir daí, os dados mantiveram-se constantes.

\section{Conclusão}

A perda de massa corporal magra pode refletir uma alteração no metabolismo proteico durante o pós-operatório imediato da cirurgia bariátrica, que pode implicar em evolução clínica e nutricional desfavoráveis.

Termos de indexação: Cirurgia bariátrica. Composição corporal. Impedância elétrica. Nutrição em saúde pública. Perda de peso.

\footnotetext{
1 Universidade de São Paulo, Faculdade de Medicina de Ribeirão Preto, Curso de Nutrição e Metabolismo. Av. Bandeirantes 3900, Monte Alegre, 14049-900, Ribeirão Preto, SP, Brasil. Correspondência para/Correspondence to: S.F.C. CUNHA. E-mail: <sfreire@fmrp.usp.br>.
} 
536 | S.F.C. CUNHA et al.

\section{A B S T R A C T}

\section{Objective}

The aim of this study was to determine changes in lean body mass after bariatric surgery.

\section{Methods}

This retrospective study reviewed 17 medical records of obese women who underwent banded Roux-en-Y gastric bypass. The medical records contained data collected immediately before and 1, 3, 6 and 12 months after surgery. The data included age, weight, height and lean and fat body mass determined by bioelectrical impedance analysis.

\section{Results}

The mean age of the patients was 43.1 years (SD=7.7). Body mass index decreased significantly within 12 months of the surgery, going from $51.2(40.2-74.1)$ to $33.7(24.8-53.4) \mathrm{kg} / \mathrm{m}^{2}$, as did fat body mass, going from $67.5(51.2-67.4)$ to $32.1(16.4-61.9) \mathrm{kg}$. In the first month after surgery, lean body mass decreased from $M=65.3(S D=7.6)$ to $M=59.7(S D=8.1 \mathrm{~kg})$, representing a decrease of $8.5 \%$. Lean body mass remained constant after this period.

\section{Conclusion}

Loss of lean body mass may indicate a change in protein metabolism immediately after bariatric surgery, which may result in an unfavorable clinical and nutritional course.

Indexing terms: Bariatric surgery. Body composition. Electric impedance. Nutrition, public health. Weight loss.

\section{N T R O D U ÇÃ O}

O tratamento cirúrgico da obesidade, denominado cirurgia bariátrica, pode utilizar técnicas disabsortivas, restritivas ou mistas. Nas cirurgias disabsortivas, são realizadas derivações no intestino delgado, implicando em perda de peso pela limitação na absorção de nutrientes. A redução importante do volume gástrico, por gastroplastia parcial e/ou colocação de banda gástrica, caracterizam as cirurgias restritivas ${ }^{1}$. Estas técnicas promovem a saciedade precoce e dificultam o consumo rápido de alimentos, restringido o volume ingerido. A Derivação Gástrica em $Y$ de Roux (DGYR) com anel de contenção gástrica é um exemplo de técnica cirúrgica mista, sendo realizada a redução no volume gástrico associada à derivação do intestino delgado².

Dentre os pacientes submetidos ao tratamento cirúrgico da obesidade, 70\% mantém perda de peso por cinco anos, que resulta em melhora da função cardiovascular, do perfil lipídico, da apneia do sono, da atividade física e da habilidade de trabalho ${ }^{3}$. Entretanto, o pós-operatório pode cursar com complicações cirúrgicas, psicológicas ou nutricionais, que devem ser identificadas precocemente pela equipe multiprofissional. A avaliação e o seguimento nutricional visam identificar os pacientes com evolução desfavorável na perda de peso e diagnosticar e intervir nos distúrbios nutricionais.

Os métodos de avaliação da composição corporal empregados no seguimento pós-operatório imediato e/ou tardio devem ser de fácil execução, baixo custo e não envolver procedimentos invasivos. Devido à facilidade de acesso e execução, as medidas de circunferências e a espessura de pregas cutâneas são comumente utilizadas na avaliação da evolução nutricional. Entretanto, em indivíduos obesos há dificuldade em isolar a massa gordurosa, o que diminui a precisão do método 4 .

Apesar das limitações relacionadas ao excesso de peso, a Dual-Energy X-Ray Absorptiometry (DEXA) e a pesagem hidrostática são métodos mais precisos na mensuração da porcentagem de gordura corporal ${ }^{5,6}$. A Impedância Bioelétrica (BIA) é um instrumento utilizado de rotina clínica em tais situações e baseia-se no princípio de que o fluxo elétrico é facilitado através do tecido hidra- 
tado e da água extracelular, em comparação com o tecido adiposo ${ }^{7}$, fornecendo resultados reproduzíveis e rapidamente obtidos ${ }^{8}$. O objetivo desse estudo foi determinar a perda de massa corporal magra em pacientes após cirurgia bariátrica, utilizando-se a BIA.

\section{MÉ T O D O S}

O estudo retrospectivo foi aprovado pelo Comitê de Ética em Pesquisa (processo $n^{\circ}$ 3829/2007) do Hospital das Clínicas da Faculdade de Medicina de Ribeirão Preto da Universidade de São Paulo (HC-FMRP/USP). Após exclusão dos casos com informações insuficientes, foram analisados 17 prontuários de mulheres obesas submetidas à cirurgia bariátrica pela equipe do Centro de Referência Nacional para o Tratamento Cirúrgico da Obesidade Mórbida do HC-FMRP/USP e acompanhadas ambulatorialmente em retornos periódicos. Todas as mulheres foram avaliadas por equipe multidisciplinar no pré e pós-operatório da DGYR com anel de contenção gástrica (Cirurgia de (apella), pela mesma equipe cirúrgica e utilizando-se protocolos previamente estabelecidos.

No pré-operatório e no $1^{\circ}, 6^{\circ}$ e $12^{\circ}$ retornos ambulatoriais após o procedimento cirúrgico, registrou-se o peso, a altura e os dados de composição corporal obtidos pela BIA. A estatura foi aferida por meio de haste metálica graduada com comprimento máximo de 2,0 metros e precisão de 0,5 centímetros. O peso corporal foi aferido em balança Filizola ${ }^{\circledR}$, com precisão de cem gramas, estando o indivíduo de pé, ereto, descalço e com o mínimo de roupa. O Índice de Massa Corporal (IMC) foi determinado pela relação entre o peso e a altura ao quadrado.

Para a avaliação da composição corporal, utilizou-se aparelho Quantum BIA 101q-RJL Systems (Michigan, EUA), que forneceu os valores de resistência e reactância à passagem de uma corrente elétrica de $50 \mathrm{kHz}$ por eletrodos posicionados na superfície no dorso da mão e do pé ipsilateral, estando os indivíduos em decúbito dorsal. As pacientes foram previamente orientadas a evitar consumo de álcool e não praticar atividade física extenuante no dia anterior, além de restringir a ingestão de alimentos ou líquidos quatro horas antes da realização do exame ${ }^{8}$. Através de equações de regressão linear validadas para adultos ${ }^{7}$, determinou-se a Massa Corporal Magra (MCM) e Massa Corporal Gorda (MCG) utilizando o Programa RJL Systems Weight Manager 2.05a.

Os dados foram tabulados em planilha eletrônica Excel e analisados no Programa Statistica 6.0. A normalidade da distribuição das variáveis foi testada pelo teste de Shapiro-Wilk. As variáveis com distribuição normal foram analisadas por ANOVA para medidas repetidas, seguidas pelo teste de Tukey, sendo apresentadas como Média (M) e Desvio-Padrão (DP). Variáveis sem distribuição normal foram analisadas pelo Teste de Friedman, seguidas pelo Teste de comparação múltipla para amostras dependentes, sendo apresentadas como mediana e valores mínimos e máximos. Diferenças entre as variáveis foram consideradas significativas quando $p<0,05$.

\section{RESULTA DOS}

A Tabela 1 apresenta a caracterização geral da casuística no período pré-operatório, incluindo as comorbidades associadas à obesidade, a idade, peso, altura e o IMC. Durante o período do estudo, não foram documentadas complicações relacionadas ao procedimento cirúrgico.

A cada retorno, houve diminuição significativa do IMC e da MCG, em relação aos valores obtidos no pré-operatório. A MCM diminuiu 8,5\% entre o período pré-operatório $(M=65,3$, $\mathrm{DP}=7,6 \mathrm{~kg})$ e o $1^{\circ}$ mês após a cirurgia $(M=59,7$, $D P=8,1 \mathrm{~kg}$ ), sendo que a partir daí, os valores mantiveram-se constantes. A redução da MCM no $1^{\circ}$ mês ocorreu em $47,1 \%$ das mulheres (8 casos), com média de perda de 9,6kg (Tabela 2). 
538 | S.F.C. CUNHA et al.

Tabela 1. Valores individuais, média e desvio-padrão da idade, peso, altura, índice de massa corporal e comorbidades das voluntárias da pesquisa, no período pré-operatório. Ribeirão Preto (SP), 2008.

\begin{tabular}{|c|c|c|c|c|c|}
\hline Casos & Comorbidades associadas à Obesidade grau III & Idade (anos) & Peso $(\mathrm{kg})$ & Altura (m) & $\operatorname{IMC}\left(\mathrm{kg} / \mathrm{m}^{2}\right)$ \\
\hline 1 & Calculose da vesícula biliar e hiperuricemia & 53 & 107,0 & 1,45 & 50,9 \\
\hline 2 & Diabetes mellitus & 41 & 136,2 & 1,62 & 51,9 \\
\hline 3 & Sem comorbidades & 40 & 127,4 & 1,53 & 54,4 \\
\hline 4 & Hérnia ventral e umbilical & 46 & 121,4 & 1,54 & 51,2 \\
\hline 5 & Calculose da vesícula biliar & 49 & 128,2 & 1,55 & 53,4 \\
\hline 6 & Hipertrigliceridemia e hipertensão arterial sistêmica & 53 & 108,6 & 1,55 & 45,2 \\
\hline 7 & Hipertrigliceridemia, hipertensão arterial sistêmica e hérnia ventral & 36 & 156,0 & 1,57 & 63,3 \\
\hline 8 & Calculose da vesícula biliar & 48 & 182,6 & 1,57 & 74,1 \\
\hline 9 & Sem comorbidades & 34 & 124,0 & 1,63 & 46,7 \\
\hline 10 & Hipertensão arterial sistêmica & 36 & 113,5 & 1,68 & 40,2 \\
\hline 11 & Sem comorbidades & 57 & 125,8 & 1,67 & 45,1 \\
\hline 12 & Apneia do sono & 36 & 140,5 & 1,69 & 49,2 \\
\hline 13 & Sem comorbidades & 50 & 142,6 & 1,68 & 50,5 \\
\hline 14 & Sem comorbidades & 47 & 143,2 & 1,66 & 52,0 \\
\hline 15 & Sem comorbidades & 34 & 148,4 & 1,70 & 51,3 \\
\hline 16 & Hipertensão arterial sistêmica & 38 & 145,4 & 1,72 & 49,1 \\
\hline 17 & Hipertensão arterial sistêmica e calculose da vesícula biliar & 34 & 153,3 & 1,70 & 53,0 \\
\hline \multicolumn{2}{|l|}{ Média } & 43,1 & 135,5 & 1,62 & 51,9 \\
\hline \multicolumn{2}{|c|}{ Desvio-padrão } & 7,7 & 19,2 & 0,10 & 7,5 \\
\hline
\end{tabular}

IMC: índice de massa corporal.

Tabela 2. Evolução do IMC, MCM e MCG em 17 mulheres obesas submetidas à cirurgia bariátrica. Ribeirão Preto (SP), 2008.

\begin{tabular}{lcccc}
\hline & Pré-operatório & $1^{\circ}$ mês PO & $6^{\circ}$ mês PO & $12^{\circ}$ mês PO \\
\hline IMC $\left(\mathrm{kg} / \mathrm{m}^{2}\right)^{\mathbf{a}}$ & $51,2(40,2-74,1)$ & $42,7(34,5-68,6)$ & $37,1(29,6-61,3)$ & $33,7(24,8-53,4)$ \\
MCM (kg) & $\mathrm{M}=65,3 \mathrm{DP}=7,6$ & $\mathrm{M}=59,7, \mathrm{DP}=8,1$ & $\mathrm{M}=58,5, \mathrm{DP}=7,2$ & $\mathrm{M}=58,4, \mathrm{DP}=6,8$ \\
$\mathrm{MCG}(\mathrm{kg})^{\mathbf{a}}$ & $67,5(51,2-67,4)$ & $49,5(32,0-98,4)$ & $39,8(27,3-76,3)$ & $32,1(16,4-61,9)$ \\
\hline
\end{tabular}

${ }^{\mathbf{a}}$ existe diferença estatística entre todos os período de estudo; ${ }^{\mathbf{b}}$ existe diferença estatística apenas entre o pré-operatório e o $1^{\circ}$ mês do PO IMC: índice de massa corporal; MCM: massa corporal magra; MCG: massa corporal gorda; PO: pós-operatório.

Na comparação entre o IMC e MCG nas diferentes ocasiões do estudo, utilizou-se o Teste de Friedman.

Na comparação entre a MCM nas diferentes ocasiões do estudo, utilizou-se ANOVA para medidas repetidas.

\section{DISCUSS Ã O}

Neste artigo, documentou-se diminuição do IMC e MCG em mulheres submetidas à cirurgia bariátrica, conforme expectativa do tratamento cirúrgico da obesidade. Paralelamente, houve redução de $9,6 \mathrm{~kg}$ da MCM no $1^{\circ}$ mês após a cirurgia e estabilização desses valores nos meses seguintes.

Alguns estudos têm mostrado perda substancial de massa corporal magra em pacientes submetidos à cirurgia bariátrica9-11, relacionada à evolução clínica e nutricional desfavorável. Os resultados apresentados no presente estudo foram semelhantes àqueles observados em pesquisas que utilizam diferentes métodos de avaliação da composição corporal no seguimento de pacientes submetidos à cirurgia bariátrica. Durante o seguimento de 12 meses após gastroplastia com banda vertical, 26 pacientes obesos reduziram 33\% do peso corporal, 49\% da MCG, além de $20 \%$ da MCM ( $M=76,0, D P=2,6$ vs. $M=60,8$, $\mathrm{DP}=3,4 \mathrm{~kg}$ ) avaliados pela BIA ${ }^{12}$. Madan et al. ${ }^{4}$ determinaram a composição corporal de 151 pacientes com IMC inicial de $M=48, D P=5 \mathrm{~kg} / \mathrm{m}^{2}$, que foram submetidos à bypass gástrico por via 
laparoscópica ${ }^{4}$. Ao final do $12^{\circ}$ mês do pós-operatório, a MCG diminuiu de 48,8\% para 36,7\% $(p<0,0001)$ e a água corporal total diminuiu $6,8 \mathrm{~kg}$ $(p<0,0001)$, atribuídos à menor quantidade de água corporal no pós-operatório e não às modificações da composição de massa magra e gorda. Estudos que utilizam a DEXA para avaliar a composição corporal documentaram perda de MCM na ordem de $14 \%{ }^{13}$ e 9,9\% ${ }^{14}$ em pacientes obesos após a colocação de banda gástrica. A redução de água intracelular e a manutenção dos níveis de água extracelular durante o período de perda de peso apontam para a diminuição de MCM, independente de modificações do estado de hidratação ${ }^{13}$.

Em 12 meses após cirurgia bariátrica com técnicas distintas, 19 adultos obesos (peso de 140, $8, D P=21,1 \mathrm{~kg}$ ) apresentaram perda de $50,9 \mathrm{~kg}$ do peso corporal, sendo $12,6 \mathrm{~kg}(24,8 \%)$ de MCM determinadas pela pesagem hidrostática ${ }^{9}$. Analisando dados de diluição isotópica, Benedetti et al. ${ }^{15}$ avaliaram a composição corporal de 14 indivíduos obesos antes e após trinta meses da cirurgia de derivação biliopancreática. A redução do peso corporal ocorreu nos primeiros 18 meses do pós-operatório, de substancial perda de MCG $(M=60,1, D P=13,0 \mathrm{~kg}$ vs. $M=19,0, D P=8,6 \mathrm{~kg}$, $p<0,001)$, além da diminuição significativa de $\operatorname{MCM}(M=72,5, D P=12,4$ vs $M=53,2, D P=9,1 \mathrm{~kg}$, $p<0,001)$, representando redução $25,6 \%$ dos valores iniciais. Independente da técnica cirúrgica empregada, Wadstrom et al. ${ }^{16}$ verificaram perda de 18 a $28 \%$ da gordura corporal durante os primeiros três meses do pós-operatório, seguido de uma perda contínua, embora mais lenta, de peso corporal. Ao final do $3^{\circ}$ mês, a perda de MCM foi mais acentuada nos indivíduos submetidos à colocação de banda gástrica e gastroplastia $(11,8 \mathrm{~kg})$ que aqueles com gastroplastia e banda vertical $(7,0 \mathrm{~kg})^{16}$.

Diferenças na porcentagem de perda de MCM entre estudos podem dever-se à intensidade do estresse orgânico decorrente do procedimento cirúrgico e à eventual presença de complicações associadas. Os estudos não determinam a influên- cia da intensidade da restrição nutricional na velocidade de perda de MCG e MCM. É comum que indivíduos submetidos à cirurgia bariátrica façam grandes restrições no valor energético total da dieta ${ }^{15,17}$, que aumentam a proteólise a fim de fornecer substrato para a neoglicogênese, justificando a diminuição da MCM. Da mesma forma, o grau de atividade física pode influenciar na velocidade de perda de peso corporal e na preservação da MCM. Indivíduos submetidos à derivação biliopancreática com gastrectomia longitudinal e preservação pilórica (duodenal switch) que praticavam atividade física apresentaram acréscimo de $15 \%$ da MCM, enquanto que os inativos reduziram $11 \%$ em relação aos valores iniciais ao final de um ano de seguimento ${ }^{18}$. Assim, a prática de exercícios após cirurgia bariátrica determinou maior velocidade de perda de peso corporal associada ao aumento da MCM.

A análise de células musculares obtidas pela biópsia percutânea de indivíduos obesos no pré-operatório de cirurgia bariátrica revelou maior conteúdo de proteínas por células e concentração mais baixa de potássio ${ }^{10}$. Durante o período de rápida perda de peso, houve diminuição na concentração de proteína e potássio por unidade de célula muscular e um ano após a cirurgia, a relação entre RNA/DNA permaneceu reduzida, indicando baixa taxa de síntese protéica ${ }^{10}$. Apesar de mudanças detectadas na composição da célula muscular, não têm sido documentadas modificações significativas na força da musculatura esquelética"1 e respiratória ${ }^{16}$.

Por tratar-se de estudo retrospectivo, em muitos casos, os dados dos prontuários estavam incompletos, o que determinou um número restrito de pacientes que efetivamente foram incluídos na casuística. Além disso, não há registro das informações referentes ao padrão de atividade física, embora haja informações da equipe multidisciplinar que todas as voluntárias exerciam apenas as atividades laborais habituais.

Variações no estado hidroeletrolítico, distribuição de fluidos corporais e excesso de tecido adiposo são limitações da BIA ${ }^{19}$, que determinam 
erros na estimativa da $\mathrm{MCM}^{20,21}$. Empregando-se equações padronizadas, a MCM obtida pela BIA foi superestimada, quando comparada com os valores obtidos pela pesagem hidrostática ${ }^{22}$. Utilizando a pletismografia de deslocamento aéreo, determinou-se que a MCG é superestimada em mulheres $(1,3 \mathrm{~kg})$ e subestimada em homens $(5,6 \mathrm{~kg}, p<0,05)^{23}$. Recentemente, foram desenvolvidas equações preditivas de BIA específicas para obesos, com o objetivo de melhorar a precisão e a acurácia da quantificação da gordura corporal $^{23}$. Neste estudo, é provável que o emprego de equações padronizadas tenha superestimado a MCG das voluntárias, embora este viés possa não ser relevante, por tratar-se de análises sequenciais que utilizaram a mesma metodologia.

Concluí-se que mulheres obesas submetidas à DGYR apresentam perda considerável de MCM, o que pode refletir a inadequação da oferta energética e proteica, a inatividade física e a alteração no metabolismo proteico durante o pós-operatório imediato. A redução da MCM reflete em desordem no metabolismo proteico, que potencialmente implica em evolução clínica e nutricional desfavorável no pós-operatório da cirurgia bariátrica.

\section{COLABORADORES}

S.F.C. CUNHA contribuiu com a concepção da pesquisa, a interpretação e a contextualização dos dados. M. SANCHES, A. FARIA, C.B. NONINO-BORGES e J.E. SANTOS contribuíram com a organização das informações, a análise e a interpretação dos dados.

\section{REFERÊ NCIAS}

1. DeMaria EJ. Bariatric surgery for morbid obesity. N Engl J Med. 2007; 356(21):2176-83.

2. Cunha SFC, Machado JC, Almeida GAN. Obesidade. In: Vannucchi H, Marchini JS, editores. Nutrição clínica. Rio de Janeiro: Guanabara Koogan; 2007.

3. Sjöström L, Lindroos AK, Peltonen M, Torgerson J, Bouchard C, Carlsson B, et al. Lifestyle, diabetes and cardiovascular risk factors 10 years after bariatric surgery. N Engl J Med. 2004; 351(26): 2683-93.
4. Madan AK, Kuykendall S, Orth WS, Ternovits CA, Tichansky DS. Does laparoscopic gastric bypass result in a healthier body composition? An affirmative answer. Obes Surg. 2006; 16(4):465-8.

5. Mello MT, Dâmaso AR, Antunes HKM, Siqueira KO, Castro ML, Bertolino SV, et al. Avaliação da composição corporal em adolescentes obesos: o uso de dois diferentes métodos. Rev Bras Med Esporte. 2005; 11(5):267-70.

6. Tothill P. Dual-energy X-ray absorptiometry for the measurement of bone and soft tissue composition. Clin Nutr. 1995; 14(5):263-8.

7. Lukaski HC, Johnson PE, Bolonchuk WW, Lykken $\mathrm{Gl}$. Assessment of fat-free mass using bioeletrical impedance measurements of the human body. Am J Clin Nutr. 1985; 41(4):810-7.

8. Kyle UG, Bosaeus I, De Lorenzo A, Deurenberg P, Elia M, Gómez JM, et al. Bioelectrical impedance analysis - part II: review of principles and methods. Clin Nutr. 2004; 23(6):1430-53.

9. Carey DG, Pliego GJ, Raymond RL. Body composition and metabolic changes following bariatric surgery: effects on fat mass lean mass and basal metabolic rate: six months to one-year follow-up. Obes Surg. 2006; 16(12):1602-8.

10. Wadstrom C, Backman L, Forsberg AM, Nilsson E, Hultman $\mathrm{E}$, Reizenstein $\mathrm{P}$, et al. Body composition and muscle constituents during weight loss: studies in obese patients following gastroplasty. Obes Surg. 2000; 10(3):203-13.

11. Wadstrom C, Larsson L, Knutsson E, Edstrom L. The effect of excessive weight loss on skeletal muscle in man. A study of obese patients following gastroplasty. Eur J Surg. 1991; 157(5):347-54.

12. Gahtan V, Goode SE, Kurto HZ, Schocken DD, Powers P, Rosemurgy AS. Body composition and source of weight loss after bariatric surgery. Obes Surg. 1997; 7(3):184-8.

13. Sergi G, Lupoli L, Busetto L, Volpato S, Coin A, Bertani $R$, et al. Changes in fluid compartments and body composition in obese women after weight loss induced by gastric banding. Ann Nutr Metab. 2003; 47(3-4):152-7.

14. Garrapa GG, Canibus P, Gatti C, Santangelo M, Frezza F, Feliciotti $F$, et al. Changes in body composition and insulin sensitivity in severely obese subjects after laparoscopic adjustable silicone gastric banding (LASGB). Med Sci Monit. 2005; 11(11):CR522-8.

15. Benedetti G, Mingrone G, Marcoccia S, Benedetti M, Giancaterini A, Greco AV, et al. Body composition and energy expenditure after weight loss following bariatric surgery. J Am Coll Nutr. 2000; 19(2): 270-74. 
16. Wadstrom C, Muller-Suur R, Backman L. Influence of excessive weight loss on respiratory function. A study of obese patients following gastroplasty. Eur J Surg. 1991; 157(5):341-6.

17. Bortoluzzo RF. Evolução nutricional e práticas alimentares de obesos mórbidos submetidos à cirurgia bariátrica em um hospital da rede pública [dissertação]. São Paulo: Universidade de São Paulo; 2005.

18. Metcalf B, Rabkin RA, Rabkin JM, Metcalf LJ, Lehman-Becker LB. Weight loss composition: the effects of exercise following obesity surgery as measured by bioelectrical impedance analysis. Obes Surg. 2005; 15(3):183- 6.

19. Thomas BJ, Ward LC, Cornish BH. Bioimpedance spectrometry in the determination of body water compartments: accuracy and clinical significance. Appl Radiat Isto. 1998; 49(5-6):447-55.

20. Fijter CW, Fijter MM, Oe LP, Donker AJ, Vries PM. The impact of hydration status on the assessment of lean body mass by body electrical impedance in dialysis patients. Adv Perit Dial. 1993; 9:101-4.

21. Kamimura MA, Draibe AS, Sigulem DM, Cuppari L. Métodos de avaliação da composição corporal em pacientes submetidos à hemodiálise. Rev Nutr. 2004; 17(1):97-105. doi: 10.1590/\$1415-52732004 000100011.

22. Gray DS, Bray GA, Gemayel N, Kaplan K. Effect of obesity on bioelectrical impedance. Am J Clin Nutr. 1989; 50(2):255-60.

23. Horie LM, Barbosa-Silva MC, Torrinhas RS, de Mello MT, Cecconello I, Waitzberg DL. New body fat prediction equations for severely obese patients. Clin Nutr. 2008; 27(3):350-6.

Recebido em: 29/7/2008

Versão final reapresentada em: 30/10/2009 Aprovado em: 19/2/2010 